CRYSTALLOGRAPHIC COMMUNICATIONS

ISSN 2056-9890

Received 8 September 2015

Accepted 6 October 2015

Edited by G. Smith, Queensland University of Technology, Australia

Keywords: barbiturates; crystal structure; hydrogen bonding; isostructurality; topology; $X P a c$

CCDC reference: 1429681

Supporting information: this article has supporting information at journals.iucr.org/e

\section{Crystal structure of 5-hydroxy-5-propylbarbituric acid}

\author{
Thomas Gelbrich* and Ulrich J. Griesser
}

University of Innsbruck, Institute of Pharmacy, Innrain 52, 6020 Innsbruck, Austria. *Correspondence e-mail: thomas.gelbrich@uibk.ac.at

Molecules of the title compound, $\mathrm{C}_{7} \mathrm{H}_{10} \mathrm{~N}_{2} \mathrm{O}_{4}$, systematic name 5-hydroxy-5propylpyrimidine-2,4,6(1H,3H,5H)-trione, form a hydrogen-bonded framework which is based on three independent hydrogen bonds, $\mathrm{N}-\mathrm{H} \cdots \mathrm{O}$ (carbonyl), $\mathrm{N}-\mathrm{H} \cdots \mathrm{O}$ (hydroxy) and $\mathrm{O}-\mathrm{H} \cdots \mathrm{O}$ (carbonyl). This framework has the topology of the 5-connected nov net. Each molecule is linked to five other molecules via six hydrogen bonds, and the descriptor of the hydrogen-bonded structure is $F 6_{5}\left[4^{4} \cdot 6^{6}\right.$-nov]. The crystal packing is isostructural with that of the previously reported 5-hydroxy-5-ethyl analogue.

\section{Chemical context}

As part of a systematic investigation of solid-state properties of derivatives of barbituric acid (Gelbrich et al., 2015; Zencirci et al., 2014; Rossi et al., 2012), we are studying the polymorphism of a group of 5-monosubstituted barbituric acids. The title compound is an oxidation product of 5-propylbarbituric acid, formed during a crystallization experiment and the structure is reported herein. The analogous oxidation product of 5-ethylbarbituric acid was previously reported by Gatehouse \& Craven (1971).<smiles>CCCC1(O)C(=O)NC(=O)NC1=O</smiles>

\section{Structural commentary}

The molecule of the title compound (Fig. 1) displays a pyrimidine ring $(\mathrm{N} 1 / \mathrm{C} 2 / \mathrm{N} 3 / \mathrm{C} 4 / \mathrm{C} 5 / \mathrm{C} 6)$ in a C5-envelope conformation. The ring puckering parameters calculated with PLATON (Spek, 2009) are $\theta=134.4$ (3), $\Phi=52.2(5)^{\circ}$ and $Q=$ 0.2420 (14) $\AA$. The distance of C5 from the mean plane defined by the other four ring atoms [maximum deviation: N3; -0.033 (1) $\AA$ ] is -0.342 (2) $\AA$. At ring atom C5 the propyl substituent adopts a trans conformation, and the corresponding torsion angle $\mathrm{C} 5-\mathrm{C} 8-\mathrm{C} 9-\mathrm{C} 10$ is $-164.80(13)^{\circ}$. The $\mathrm{C} 5-\mathrm{C} 8-\mathrm{C} 9-\mathrm{C} 10$ fragment is twisted significantly out of the plane defined by atoms $\mathrm{C} 8, \mathrm{C} 5$ and $\mathrm{C} 2$, which bisects the pyrimidinetrione fragment into two approximately symmetrical halves, resulting in a pseudo-torsion angle $\mathrm{C} 2 \cdots \mathrm{C} 5-$ 


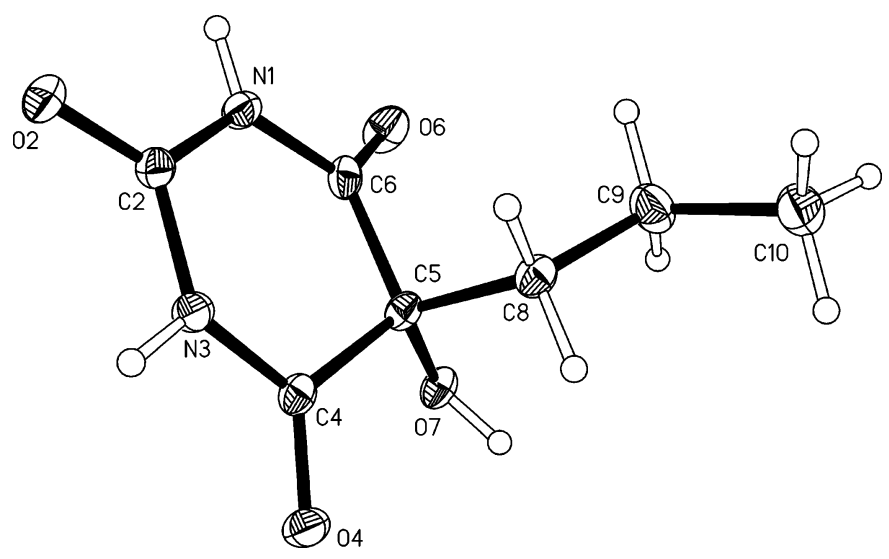

Figure 1

Asymmetric unit with displacement ellipsoids drawn at the $50 \%$ probability level and hydrogen atoms drawn as spheres of arbitrary size.

C8-C9 of $-125.69(11)^{\circ}$. Closer inspection suggests that this particular geometry may help to prevent unfavourably close intramolecular contacts between the $\mathrm{O} 7$ hydroxy group and the $\mathrm{CH}_{2}$ group at $\mathrm{C}$, and may be also facilitate the participation of the hydroxy group in complex intermolecular hydrogen-bonding interactions.

\section{Supramolecular features}

One NH group and one carbonyl group of the molecule are engaged in a centrosymmetric two-point interaction, N3$\mathrm{H} 3 \cdots \mathrm{O} 4^{\mathrm{ii}}$ (Table 1), resulting in an $R_{2}^{2}(8)$ ring (Etter et al., 1990; Bernstein et al., 1995). This kind of ring is a ubiquitous feature in crystal structures of barbiturates (Gelbrich et al., 2011). The other NH group is bonded to the hydroxy group of a second molecule via a 2 operation, $\mathrm{N} 1-\mathrm{H} 1 \cdots \mathrm{O} 7^{\mathrm{i}}$, and this interaction is accompanied by a short $\mathrm{O} 6 \cdot \mathrm{C}^{\mathrm{i}}$ contact [2.8654 (18) ̊]. Additionally, the hydroxy group donates a

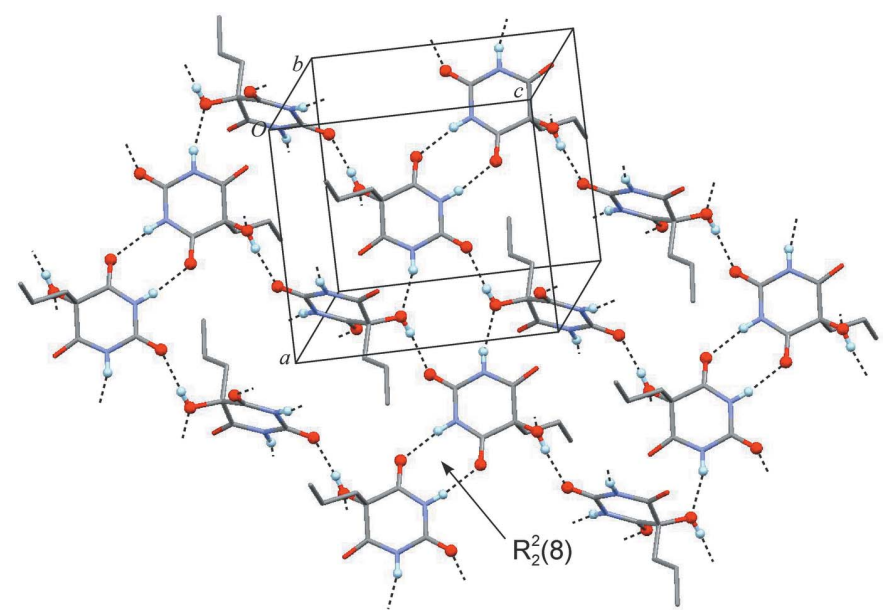

Figure 2

Layer fragment of the H-bonded framework which contains rings connecting four and six molecules in addition to $R_{2}^{2}(8)$ rings. Hydrogen bonds are drawn as dashed lines. $\mathrm{H}$ and $\mathrm{O}$ atoms engaged in hydrogen bonding are drawn as balls and all the other $\mathrm{H}$ atoms are omitted for clarity.
Table 1

Hydrogen-bond geometry $\left(\AA{ }^{\circ}\right)$.

\begin{tabular}{lllll}
\hline$D-\mathrm{H} \cdots A$ & $D-\mathrm{H}$ & $\mathrm{H} \cdots A$ & $D \cdots A$ & $D-\mathrm{H} \cdots A$ \\
\hline $\mathrm{N} 1-\mathrm{H} 1 \cdots \mathrm{O} 7^{\mathrm{i}}$ & $0.87(1)$ & $2.03(1)$ & $2.8683(17)$ & $164(2)$ \\
$\mathrm{N} 3-\mathrm{H} 3 \cdots \mathrm{O} 4^{\mathrm{ii}}$ & $0.86(1)$ & $2.00(1)$ & $2.8451(16)$ & $170(2)$ \\
$\mathrm{O} 7-\mathrm{H} 7 \cdots 2^{\mathrm{iii}}$ & $0.84(2)$ & $1.98(2)$ & $2.8055(15)$ & $169(2)$ \\
\hline $\begin{array}{l}\text { Symmetry codes: } \\
x-\frac{1}{2},-y+\frac{3}{2}, z-\frac{1}{2} .\end{array}$ & & & &
\end{tabular}

hydrogen bond to the $\mathrm{C} 2$ carbonyl group of another molecule related by glide symmetry $\left(\mathrm{O} 7-\mathrm{H} 7 \cdots \mathrm{O} 2^{\mathrm{iii}}\right)$. Altogether, six hydrogen bonds connect each molecule to five other molecules. In addition to the aforementioned $R_{2}^{2}(8)$ rings, the resulting hydrogen-bonded framework structure also displays rings composed of four and six molecules (Fig. 2). This 5connected framework has the topology of the nov structure (Blatov et al., 2004). Fig. 3 shows a graph of the hydrogenbonded structure (HBS) according to the methodology proposed by Hursthouse et al. (2015). The short descriptor according to Hursthouse et al. (2015) for this HBS is $F 6_{5}\left[4^{4} .6^{6}\right.$ nov].

\section{Database survey}

The Cambridge Structural Database (Version 5.36; Groom \& Allen, 2014) contains the crystal structure of 5,5-dihydroxybarbituric acid (Singh, 1965; Harrowfield et al., 1989; CSD refcode ALXANM01) and those of a monohydrate (Lewis \& Tocher, 2004; PAGYUS), a trihydrate (Lewis \& Tocher, 2004b; HBARBT01) and a 1,4-dioxane hemisolvate (Gelbrich et al., 2010; NUQYII) of the same compound. Two-point

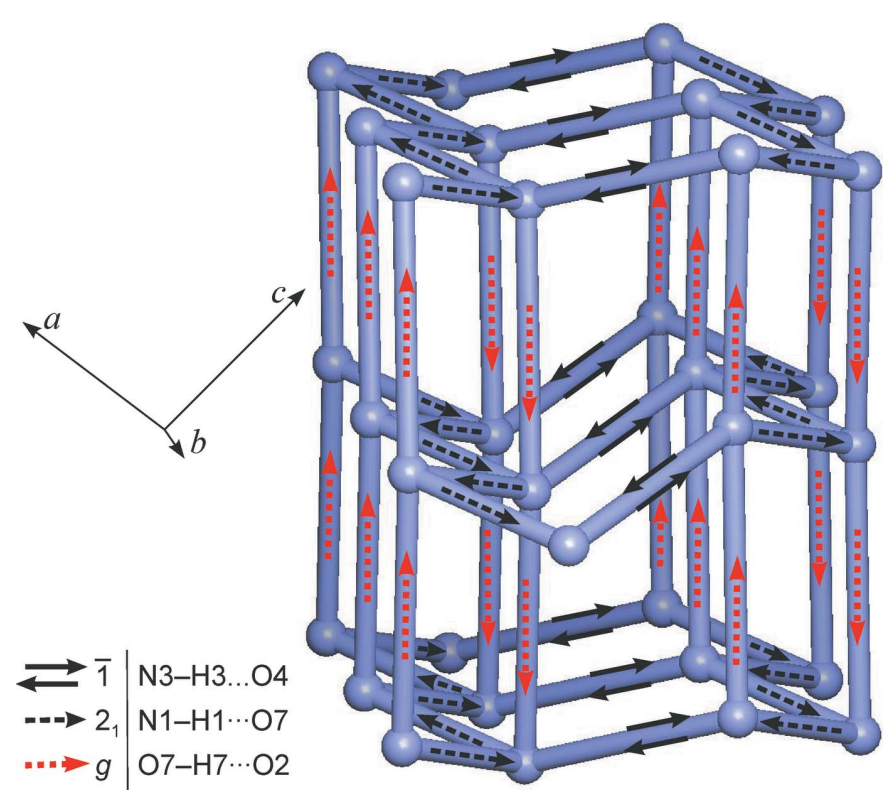

Figure 3

The $\mathrm{N}-\mathrm{H} \cdots \mathrm{O}$ (carbonyl), $\mathrm{N}-\mathrm{H} \cdots \mathrm{O}$ (hydroxy) and $\mathrm{O}-\mathrm{H} \cdots \mathrm{O}$ (carbonyl) bonded $F 6_{5}\left[4^{4} \cdot 6^{6}\right.$-nov $]$ structure of title compound. Molecules are represented as nodes and their hydrogen-bond connections as links between them. 
Table 2

Experimental details.

\begin{tabular}{|c|c|}
\hline \multicolumn{2}{|l|}{ Crystal data } \\
\hline Chemical formula & $\mathrm{C}_{7} \mathrm{H}_{10} \mathrm{~N}_{2} \mathrm{O}_{4}$ \\
\hline$M_{\mathrm{r}}$ & 186.17 \\
\hline Crystal system, space group & Monoclinic, $P 2_{1} / n$ \\
\hline Temperature $(\mathrm{K})$ & 100 \\
\hline$a, b, c(\AA)$ & $10.7862(8), 6.7093(5), 11.7365(6)$ \\
\hline$\beta\left(^{\circ}\right)$ & $98.632(6)$ \\
\hline$V\left(\AA^{6}\right)$ & $839.72(10)$ \\
\hline$Z$ & 4 \\
\hline Radiation type & Mo $K \alpha$ \\
\hline$\mu\left(\mathrm{mm}^{-1}\right)$ & 0.12 \\
\hline Crystal size (mm) & $0.05 \times 0.05 \times 0.05$ \\
\hline \multicolumn{2}{|l|}{ Data collection } \\
\hline Diffractometer & Rigaku Saturn724+ \\
\hline Absorption correction & $\begin{array}{l}\text { Multi-scan (CrysAlis PRO; } \\
\quad \text { Agilent, 2014) }\end{array}$ \\
\hline$T_{\min }, T_{\max }$ & $0.809,1.000$ \\
\hline $\begin{array}{l}\text { No. of measured, independent and } \\
\text { observed }[I>2 \sigma(I)] \text { reflections }\end{array}$ & $5354,1724,1354$ \\
\hline$R_{\text {int }}$ & 0.034 \\
\hline$(\sin \theta / \lambda)_{\max }\left(\AA^{-1}\right)$ & 0.625 \\
\hline \multicolumn{2}{|l|}{ Refinement } \\
\hline$R\left[F^{2}>2 \sigma\left(F^{2}\right)\right], w R\left(F^{2}\right), S$ & $0.037,0.095,1.05$ \\
\hline No. of reflections & 1724 \\
\hline No. of parameters & 138 \\
\hline No. of restraints & 2 \\
\hline $\mathrm{H}$-atom treatment & $\begin{array}{l}\mathrm{H} \text { atoms treated by a mixture of } \\
\text { independent and constrained } \\
\text { refinement }\end{array}$ \\
\hline$\Delta \rho_{\max }, \Delta \rho_{\min }\left(\mathrm{e} \AA^{-3}\right)$ & $0.29,-0.20$ \\
\hline
\end{tabular}

Computer programs: CrystalClear-SM Expert (Rigaku, 2012), CrysAlis PRO (Agilent, 2014), SHELXT (Sheldrick, 2015a), SHELXL2014/6 (Sheldrick, 2015b), XP in SHELXTL (Sheldrick, 20088) and Mercury (Macrae et al., 2006) and publCIF (Westrip, 2010).

connections based on $\mathrm{N}-\mathrm{H} \cdots \mathrm{O}=\mathrm{C}$ bonds which result in characteristic $R_{2}^{2}(8)$ rings are found in each of these compounds.

The title structure displays just one such interaction which involves the carbonyl group at ring position 4 (Fig. 4). One such connection, albeit via the $\mathrm{C} 2$ carbonyl group, also exists in the 5,5-dihydroxybarbituric acid structure. Here it forms part of the $C$-4 ladder motif which is known from 5,5-disubstituted derivatives of barbituric acid (Gelbrich et al., 2011).

The monohydrate and 1,4-dioxane hemisolvate each contain two two-point $\mathrm{N}-\mathrm{H} \cdots \mathrm{O}=\mathrm{C}$ connections per molecule, in the first case via the topologically equivalent $\mathrm{C} 4$ and C6 carbonyl groups and in the second via the $\mathrm{C} 4$ and $\mathrm{C} 2$ carbonyl groups, resulting in the looped chain motifs $C-2$ and $C-1$ (Gelbrich et al., 2011), respectively, which are frequently encountered in barbiturates. $C-2$ chains are also found in the structure of the trihydrate. The molecular conformation of 5hydroxy-5-ethylbarbituric acid (Gatehouse \& Craven, 1971; HEBARB) is similar to that of the title structure with respect to the pseudo-torsion angle of $124.3^{\circ}$, which is structurally analogous to the $\mathrm{C} 2 \cdots \mathrm{C} 5-\mathrm{C} 8-\mathrm{C} 9$ angle discussed above. A comparison with the program XPac (for details, see below) indicated that these two compounds are indeed isostructural. Geometrical differences between the two molecular packing arrangements are small (Fig. 4), which is reflected in a calculated $\mathrm{XPac}$ dissimilarity index of just 5.4. This close packing similarity is remarkable insofar as the substitution of a propyl with an ethyl group alters the molecular shape considerably and leads to an $11 \%$ decrease in the volume of the unit cell. The unit-cell parameters of the two isostructures correspond directly with one another. The $a$ and $b$ axes of the ethyl analogue (determined at room temperature) are $6.1 \%$ and $6.5 \%$ shorter than those of the title compound. Simultaneously, the $c$ axis of the ethyl analogue is $1.5 \%$ longer and the $\beta$ angle is enlarged by $1.0^{\circ}$.

\section{Synthesis and crystallization}

A glass slide with a sample of 5-propylbarbituric acid embedded in paraffin oil was placed on a hot bench. The sample was melted and left to crystallize. Within a few days, the original crystals had partially converted and cube-shaped single crystals of the title compound had formed.
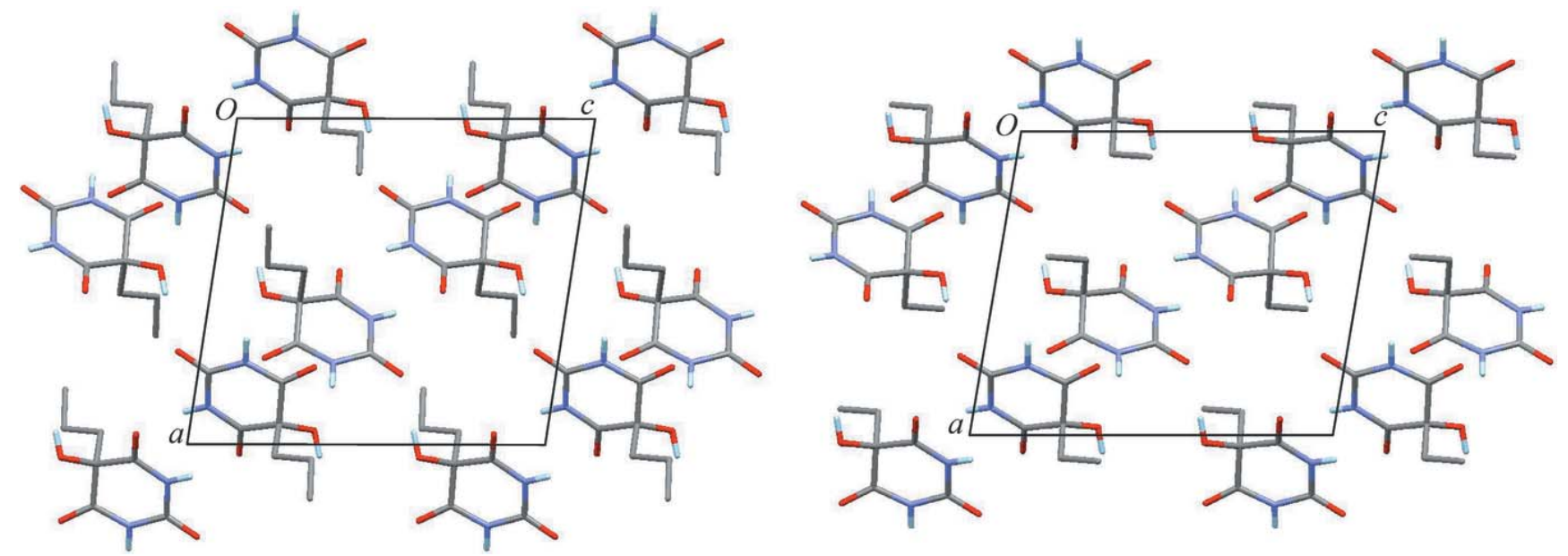

Figure 4

An illustration of the similar packing of molecules in the title compound (left) and its ethyl analogue (right). Each structure is viewed along its [010] direction. $\mathrm{H}$ atoms in alkyl groups are omitted for clarity. 


\section{Refinement}

Crystal data, data collection and structure refinement details are summarised in Table 2. The data collection was carried out in the manner described by Coles \& Gale (2012). All H atoms were identified in difference maps. Methyl $\mathrm{H}$ atoms were idealized and included as rigid groups allowed to rotate but not tip $\left(\mathrm{C}-\mathrm{H}=0.98 \AA\right.$ ). $\mathrm{H}$ atoms bonded to secondary $\mathrm{CH}_{2}$ carbon atoms were positioned geometrically $(\mathrm{C}-\mathrm{H}=0.99 \AA)$. Hydrogen atoms bonded to $\mathrm{N}$ atoms were refined with restrained distances $[\mathrm{N}-\mathrm{H}=0.86$ (1) $\AA$ ] . The hydrogen atom of the hydroxy group was refined freely and the $U_{\text {iso }}$ parameters of all hydrogen atoms were also refined freely.

\section{Analysis of structural features}

The topology of the HBS was determined and classified with the programs ADS and IsoTest of the TOPOS package (Blatov, 2006) in the manner described by Baburin \& Blatov (2007). The topology graph for the HBS (Fig. 3) is based on a net drawn with the IsoCryst program of the TOPOS package. The HBS of the title structure was defined by the three interactions $\mathrm{N}-\mathrm{H} \cdots \mathrm{O}$ (carbonyl), $\mathrm{N}-\mathrm{H} \cdots \mathrm{O}$ (hydroxy) and $\mathrm{O}-\mathrm{H} \cdots \mathrm{O}$ (carbonyl) listed in Table 1 . The molecular packing in the title compound and its ethyl analogue were compared using the program XPac (Gelbrich \& Hursthouse, 2005). The underlying calculations were based on a comparison of sets of intermolecular geometrical parameters generated from all non-H atomic positions of the title compound, except for the methyl carbon atom, and all 12 non-H atomic positions of the ethyl analogue. A match of two complete clusters consisting of a central molecule and 17 coordinating molecules was obtained with a dissimilarity index (Gelbrich et al., 2012) of 5.4, indicating isostructurality of the two compounds with a high degree of packing similarity.

\section{Acknowledgements}

We thank Professor Simon Coles (Southampton) for access to the diffractometer used in this study.

\section{References}

Agilent (2014). CrysAlis PRO. Agilent Technologies, Yarnton, England.

Baburin, I. A. \& Blatov, V. A. (2007). Acta Cryst. B63, 791-802.

Bernstein, J., Davis, R. E., Shimoni, L. \& Chang, N.-L. (1995). Angew. Chem. Int. Ed. Engl. 34, 1555-1573.

Blatov, V. A. (2006). IUCr Comput. Comm. Newsl. 7, 4-38.

Blatov, V. A., Carlucci, L., Ciani, G. \& Proserpio, D. M. (2004). CrystEngComm, 6, 378-395.

Coles, S. J. \& Gale, P. A. (2012). Chem. Sci. 3, 683-689.

Etter, M. C., MacDonald, J. C. \& Bernstein, J. (1990). Acta Cryst. B46, 256-262.

Gatehouse, B. M. \& Craven, B. M. (1971). Acta Cryst. B27, 1337-1344.

Gelbrich, T. \& Hursthouse, M. B. (2005). CrystEngComm, 7, 324336.

Gelbrich, T., Meischberger, I. \& Griesser, U. J. (2015). Acta Cryst. C71, 204-210.

Gelbrich, T., Rossi, D. \& Griesser, U. J. (2010). Acta Cryst. E66, o1219.

Gelbrich, T., Rossi, D., Häfele, C. A. \& Griesser, U. J. (2011). CrystEngComm, 13, 5502-5509.

Gelbrich, T., Threlfall, T. L. \& Hursthouse, M. B. (2012). CrystEngComm, 14, 5454-5464.

Groom, C. R. \& Allen, F. H. (2014). Angew. Chem. Int. Ed. 53, 662671.

Harrowfield, J. M., Skelton, B. W., Soudi, A. A. \& White, A. H. (1989). Aust. J. Chem. 42, 1795-1798.

Hursthouse, M. B., Hughes, D. S., Gelbrich, T. \& Threlfall, T. L. (2015). Chem. Cent. J. 9, 1.

Lewis, T. C. \& Tocher, D. A. (2004a). Acta Cryst. E60, o1689-o1690.

Lewis, T. C. \& Tocher, D. A. (2004b). Acta Cryst. E60, o1748-o1750.

Macrae, C. F., Edgington, P. R., McCabe, P., Pidcock, E., Shields, G. P., Taylor, R., Towler, M. \& van de Streek, J. (2006). J. Appl. Cryst. 39, 453-457.

Rigaku (2012). CrystalClear-SM Expert. Rigaku/MSC Inc., The Woodlands, Texas, USA.

Rossi, D., Gelbrich, T., Kahlenberg, V. \& Griesser, U. J. (2012). CrystEngComm, 14, 2494-2506.

Sheldrick, G. M. (2008). Acta Cryst. A64, 112-122.

Sheldrick, G. M. (2015a). Acta Cryst. A71, 3-8.

Sheldrick, G. M. (2015b). Acta Cryst. C71, 3-8.

Singh, C. (1965). Acta Cryst. 19, 759-767.

Spek, A. L. (2009). Acta Cryst. D65, 148-155.

Westrip, S. P. (2010). J. Appl. Cryst. 43, 920-925.

Zencirci, N., Griesser, U. J., Gelbrich, T., Kahlenberg, V., Jetti, R. K. R., Apperley, D. C. \& Harris, R. K. (2014). J. Phys. Chem. B, 118, 3267-3280. 


\section{supporting information}

Acta Cryst. (2015). E71, 1311-1314 [https://doi.org/10.1107/S2056989015018769]

\section{Crystal structure of 5-hydroxy-5-propylbarbituric acid}

\section{Thomas Gelbrich and Ulrich J. Griesser}

\section{Computing details}

Data collection: CrystalClear-SM Expert (Rigaku, 2012); cell refinement: CrysAlis PRO (Agilent, 2014); data reduction: CrysAlis PRO (Agilent, 2014); program(s) used to solve structure: SHELXT (Sheldrick, 2015a); program(s) used to refine structure: SHELXL2014/6 (Sheldrick, 2015b); molecular graphics: XP in SHELXTL (Sheldrick, 20088) and Mercury

(Macrae et al., 2006); software used to prepare material for publication: publCIF (Westrip, 2010).

5-Hydroxy-5-propylpyrimidine-2,4,6(1H,3H),5H)-trione

Crystal data

$\mathrm{C}_{7} \mathrm{H}_{10} \mathrm{~N}_{2} \mathrm{O}_{4}$

$M_{r}=186.17$

Monoclinic, $P 22_{1} / n$

$a=10.7862(8) \AA$

$b=6.7093(5) \AA$

$c=11.7365(6) \AA$

$\beta=98.632(6)^{\circ}$

$V=839.72(10) \AA^{3}$

$Z=4$

Data collection

Rigaku Saturn724+ diffractometer

Radiation source: Sealed Tube

Graphite Monochromator monochromator Detector resolution: 28.5714 pixels $\mathrm{mm}^{-1}$ profile data from $\omega$-scans Absorption correction: multi-scan

(CrysAlisPro; Agilent, 2014)

$T_{\min }=0.809, T_{\max }=1.000$

\section{Refinement}

Refinement on $F^{2}$

Least-squares matrix: full

$R\left[F^{2}>2 \sigma\left(F^{2}\right)\right]=0.037$

$w R\left(F^{2}\right)=0.095$

$S=1.05$

1724 reflections

138 parameters

2 restraints

Primary atom site location: structure-invariant direct methods
$F(000)=392$

$D_{\mathrm{x}}=1.473 \mathrm{Mg} \mathrm{m}^{-3}$

Mo $K \alpha$ radiation, $\lambda=0.71073 \AA$

Cell parameters from 3013 reflections

$\theta=2.4-27.5^{\circ}$

$\mu=0.12 \mathrm{~mm}^{-1}$

$T=100 \mathrm{~K}$

Cube, colourless

$0.05 \times 0.05 \times 0.05 \mathrm{~mm}$

5354 measured reflections

1724 independent reflections

1354 reflections with $I>2 \sigma(I)$

$R_{\text {int }}=0.034$

$\theta_{\max }=26.4^{\circ}, \theta_{\min }=2.4^{\circ}$

$h=-12 \rightarrow 13$

$k=-8 \rightarrow 8$

$l=-14 \rightarrow 14$

Secondary atom site location: difference Fourier map

Hydrogen site location: mixed

$\mathrm{H}$ atoms treated by a mixture of independent and constrained refinement

$w=1 /\left[\sigma^{2}\left(F_{\mathrm{o}}^{2}\right)+(0.0498 P)^{2}+0.1441 P\right]$ where $P=\left(F_{\mathrm{o}}{ }^{2}+2 F_{\mathrm{c}}{ }^{2}\right) / 3$

$(\Delta / \sigma)_{\max }<0.001$

$\Delta \rho_{\max }=0.29 \mathrm{e} \AA^{-3}$

$\Delta \rho_{\min }=-0.20$ e $\AA^{-3}$ 


\section{Special details}

Geometry. All e.s.d.'s (except the e.s.d. in the dihedral angle between two 1.s. planes) are estimated using the full covariance matrix. The cell e.s.d.'s are taken into account individually in the estimation of e.s.d.'s in distances, angles and torsion angles; correlations between e.s.d.'s in cell parameters are only used when they are defined by crystal symmetry. An approximate (isotropic) treatment of cell e.s.d.'s is used for estimating e.s.d.'s involving l.s. planes.

Fractional atomic coordinates and isotropic or equivalent isotropic displacement parameters $\left(\hat{A}^{2}\right)$

\begin{tabular}{lllll}
\hline & $x$ & $y$ & $z$ & $U_{\text {iso }} / U_{\text {eq }}$ \\
\hline N1 & $0.75066(12)$ & $0.60615(19)$ & $0.37252(9)$ & $0.0154(3)$ \\
H1 & $0.8189(12)$ & $0.536(3)$ & $0.3792(16)$ & $0.033(5)^{*}$ \\
O2 & $0.78456(10)$ & $0.66277(16)$ & $0.56534(8)$ & $0.0201(3)$ \\
C2 & $0.72112(13)$ & $0.6895(2)$ & $0.47207(11)$ & $0.0147(3)$ \\
N3 & $0.61584(11)$ & $0.80699(19)$ & $0.46014(9)$ & $0.0150(3)$ \\
H3 & $0.5995(14)$ & $0.870(2)$ & $0.5200(10)$ & $0.020(4)^{*}$ \\
O4 & $0.47047(9)$ & $0.99725(16)$ & $0.35153(8)$ & $0.0180(3)$ \\
C4 & $0.54700(13)$ & $0.8640(2)$ & $0.35743(11)$ & $0.0139(3)$ \\
C5 & $0.56139(13)$ & $0.7360(2)$ & $0.25307(11)$ & $0.0140(3)$ \\
O6 & $0.73523(10)$ & $0.58567(18)$ & $0.17883(8)$ & $0.0220(3)$ \\
C6 & $0.68995(13)$ & $0.6406(2)$ & $0.26184(11)$ & $0.0153(3)$ \\
O7 & $0.54144(10)$ & $0.85117(16)$ & $0.15172(8)$ & $0.0161(3)$ \\
H7 & $0.465(2)$ & $0.864(3)$ & $0.1270(16)$ & $0.040(6)^{*}$ \\
C8 & $0.46287(14)$ & $0.5674(2)$ & $0.25253(11)$ & $0.0158(3)$ \\
H8A & 0.4849 & 0.4858 & 0.3229 & $0.021(4)^{*}$ \\
H8B & 0.3799 & 0.6284 & 0.2557 & $0.016(4)^{*}$ \\
C9 & $0.45248(15)$ & $0.4312(2)$ & $0.14778(13)$ & $0.0225(4)$ \\
H9A & 0.4526 & 0.5128 & 0.0775 & $0.035(5)^{*}$ \\
H9B & 0.5262 & 0.3417 & 0.1553 & $0.033(5)^{*}$ \\
C10 & $0.33380(15)$ & $0.3070(3)$ & $0.13545(13)$ & $0.0247(4)$ \\
H10A & 0.3383 & 0.2139 & 0.2003 & $0.034(5)^{*}$ \\
H10B & 0.3253 & 0.2319 & 0.0631 & $0.036(5)^{*}$ \\
H10C & 0.2611 & 0.3948 & 0.1350 & $0.044(6)^{*}$ \\
& & & & \\
& & & &
\end{tabular}

Atomic displacement parameters $\left(\AA^{2}\right)$

\begin{tabular}{lllllll}
\hline & $U^{11}$ & $U^{22}$ & $U^{33}$ & $U^{12}$ & $U^{13}$ & $U^{23}$ \\
\hline $\mathrm{N} 1$ & $0.0131(7)$ & $0.0172(7)$ & $0.0154(6)$ & $0.0033(6)$ & $0.0002(5)$ & $-0.0013(5)$ \\
O2 & $0.0199(6)$ & $0.0225(6)$ & $0.0159(5)$ & $0.0037(5)$ & $-0.0043(4)$ & $-0.0010(4)$ \\
C2 & $0.0132(8)$ & $0.0136(8)$ & $0.0168(7)$ & $-0.0009(6)$ & $0.0004(5)$ & $0.0002(5)$ \\
N3 & $0.0160(7)$ & $0.0173(7)$ & $0.0114(6)$ & $0.0039(5)$ & $0.0011(5)$ & $-0.0022(5)$ \\
O4 & $0.0170(6)$ & $0.0205(6)$ & $0.0161(5)$ & $0.0058(5)$ & $0.0005(4)$ & $-0.0001(4)$ \\
C4 & $0.0110(8)$ & $0.0155(8)$ & $0.0151(7)$ & $-0.0018(6)$ & $0.0016(5)$ & $0.0005(5)$ \\
C5 & $0.0127(8)$ & $0.0169(8)$ & $0.0117(6)$ & $0.0010(6)$ & $-0.0003(5)$ & $0.0009(5)$ \\
O6 & $0.0173(6)$ & $0.0323(7)$ & $0.0168(5)$ & $0.0046(5)$ & $0.0042(4)$ & $-0.0025(5)$ \\
C6 & $0.0143(8)$ & $0.0161(8)$ & $0.0152(7)$ & $-0.0040(6)$ & $0.0009(5)$ & $-0.0003(5)$ \\
O7 & $0.0143(6)$ & $0.0202(6)$ & $0.0128(5)$ & $-0.0006(5)$ & $-0.0009(4)$ & $0.0031(4)$ \\
C8 & $0.0134(8)$ & $0.0178(8)$ & $0.0161(6)$ & $-0.0004(6)$ & $0.0021(5)$ & $0.0014(6)$ \\
C9 & $0.0226(9)$ & $0.0200(9)$ & $0.0256(8)$ & $-0.0019(7)$ & $0.0061(6)$ & $-0.0065(7)$
\end{tabular}




$\begin{array}{lllllll}\mathrm{C} 10 & 0.0262(10) & 0.0202(9) & 0.0264(8) & -0.0030(7) & -0.0002(6) & 0.0003(7)\end{array}$

Geometric parameters $(\AA, \stackrel{o}{)}$

\begin{tabular}{|c|c|c|c|}
\hline $\mathrm{N} 1-\mathrm{C} 2$ & $1.3754(18)$ & $\mathrm{O} 6-\mathrm{C} 6$ & $1.2110(17)$ \\
\hline $\mathrm{N} 1-\mathrm{C} 6$ & $1.3836(16)$ & $\mathrm{O} 7-\mathrm{H} 7$ & $0.84(2)$ \\
\hline $\mathrm{N} 1-\mathrm{H} 1$ & $0.866(9)$ & $\mathrm{C} 8-\mathrm{C} 9$ & $1.5226(19)$ \\
\hline $\mathrm{O} 2-\mathrm{C} 2$ & $1.2141(16)$ & $\mathrm{C} 8-\mathrm{H} 8 \mathrm{~A}$ & 0.9900 \\
\hline $\mathrm{C} 2-\mathrm{N} 3$ & $1.3720(19)$ & $\mathrm{C} 8-\mathrm{H} 8 \mathrm{~B}$ & 0.9900 \\
\hline N3-C4 & $1.3719(17)$ & $\mathrm{C} 9-\mathrm{C} 10$ & $1.516(2)$ \\
\hline $\mathrm{N} 3-\mathrm{H} 3$ & $0.860(9)$ & C9-H9A & 0.9900 \\
\hline $\mathrm{O} 4-\mathrm{C} 4$ & $1.2117(17)$ & C9-H9B & 0.9900 \\
\hline $\mathrm{C} 4-\mathrm{C} 5$ & $1.5228(19)$ & $\mathrm{C} 10-\mathrm{H} 10 \mathrm{~A}$ & 0.9800 \\
\hline $\mathrm{C} 5-\mathrm{O} 7$ & $1.4076(16)$ & $\mathrm{C} 10-\mathrm{H} 10 \mathrm{~B}$ & 0.9800 \\
\hline $\mathrm{C} 5-\mathrm{C} 6$ & $1.517(2)$ & $\mathrm{C} 10-\mathrm{H} 10 \mathrm{C}$ & 0.9800 \\
\hline $\mathrm{C} 5-\mathrm{C} 8$ & $1.551(2)$ & & \\
\hline $\mathrm{C} 2-\mathrm{N} 1-\mathrm{C} 6$ & $126.31(13)$ & $\mathrm{N} 1-\mathrm{C} 6-\mathrm{C} 5$ & $115.68(12)$ \\
\hline $\mathrm{C} 2-\mathrm{N} 1-\mathrm{H} 1$ & $116.4(12)$ & $\mathrm{C} 5-\mathrm{O} 7-\mathrm{H} 7$ & $111.6(14)$ \\
\hline $\mathrm{C} 6-\mathrm{N} 1-\mathrm{H} 1$ & $116.8(12)$ & $\mathrm{C} 9-\mathrm{C} 8-\mathrm{C} 5$ & $114.10(12)$ \\
\hline $\mathrm{O} 2-\mathrm{C} 2-\mathrm{N} 3$ & $121.52(13)$ & $\mathrm{C} 9-\mathrm{C} 8-\mathrm{H} 8 \mathrm{~A}$ & 108.7 \\
\hline $\mathrm{O} 2-\mathrm{C} 2-\mathrm{N} 1$ & $122.33(14)$ & $\mathrm{C} 5-\mathrm{C} 8-\mathrm{H} 8 \mathrm{~A}$ & 108.7 \\
\hline $\mathrm{N} 3-\mathrm{C} 2-\mathrm{N} 1$ & $116.15(11)$ & $\mathrm{C} 9-\mathrm{C} 8-\mathrm{H} 8 \mathrm{~B}$ & 108.7 \\
\hline $\mathrm{C} 2-\mathrm{N} 3-\mathrm{C} 4$ & $125.47(12)$ & $\mathrm{C} 5-\mathrm{C} 8-\mathrm{H} 8 \mathrm{~B}$ & 108.7 \\
\hline $\mathrm{C} 2-\mathrm{N} 3-\mathrm{H} 3$ & $117.8(10)$ & $\mathrm{H} 8 \mathrm{~A}-\mathrm{C} 8-\mathrm{H} 8 \mathrm{~B}$ & 107.6 \\
\hline $\mathrm{C} 4-\mathrm{N} 3-\mathrm{H} 3$ & $115.6(11)$ & $\mathrm{C} 10-\mathrm{C} 9-\mathrm{C} 8$ & $111.45(13)$ \\
\hline $\mathrm{O} 4-\mathrm{C} 4-\mathrm{N} 3$ & $122.02(12)$ & $\mathrm{C} 10-\mathrm{C} 9-\mathrm{H} 9 \mathrm{~A}$ & 109.3 \\
\hline $\mathrm{O} 4-\mathrm{C} 4-\mathrm{C} 5$ & $121.39(11)$ & $\mathrm{C} 8-\mathrm{C} 9-\mathrm{H} 9 \mathrm{~A}$ & 109.3 \\
\hline $\mathrm{N} 3-\mathrm{C} 4-\mathrm{C} 5$ & $116.26(13)$ & $\mathrm{C} 10-\mathrm{C} 9-\mathrm{H} 9 \mathrm{~B}$ & 109.3 \\
\hline $\mathrm{O} 7-\mathrm{C} 5-\mathrm{C} 6$ & $108.13(11)$ & $\mathrm{C} 8-\mathrm{C} 9-\mathrm{H} 9 \mathrm{~B}$ & 109.3 \\
\hline $\mathrm{O} 7-\mathrm{C} 5-\mathrm{C} 4$ & $110.43(12)$ & $\mathrm{H} 9 \mathrm{~A}-\mathrm{C} 9-\mathrm{H} 9 \mathrm{~B}$ & 108.0 \\
\hline $\mathrm{C} 6-\mathrm{C} 5-\mathrm{C} 4$ & $112.70(11)$ & $\mathrm{C} 9-\mathrm{C} 10-\mathrm{H} 10 \mathrm{~A}$ & 109.5 \\
\hline $\mathrm{O} 7-\mathrm{C} 5-\mathrm{C} 8$ & $112.27(11)$ & $\mathrm{C} 9-\mathrm{C} 10-\mathrm{H} 10 \mathrm{~B}$ & 109.5 \\
\hline $\mathrm{C} 6-\mathrm{C} 5-\mathrm{C} 8$ & $108.15(12)$ & $\mathrm{H} 10 \mathrm{~A}-\mathrm{C} 10-\mathrm{H} 10 \mathrm{~B}$ & 109.5 \\
\hline $\mathrm{C} 4-\mathrm{C} 5-\mathrm{C} 8$ & $105.19(11)$ & $\mathrm{C} 9-\mathrm{C} 10-\mathrm{H} 10 \mathrm{C}$ & 109.5 \\
\hline $\mathrm{O} 6-\mathrm{C} 6-\mathrm{N} 1$ & $120.87(14)$ & $\mathrm{H} 10 \mathrm{~A}-\mathrm{C} 10-\mathrm{H} 10 \mathrm{C}$ & 109.5 \\
\hline $\mathrm{O} 6-\mathrm{C} 6-\mathrm{C} 5$ & $123.30(12)$ & $\mathrm{H} 10 \mathrm{~B}-\mathrm{C} 10-\mathrm{H} 10 \mathrm{C}$ & 109.5 \\
\hline $\mathrm{C} 6-\mathrm{N} 1-\mathrm{C} 2-\mathrm{O} 2$ & $-174.59(14)$ & $\mathrm{C} 2-\mathrm{N} 1-\mathrm{C} 6-\mathrm{C} 5$ & $-16.4(2)$ \\
\hline $\mathrm{C} 6-\mathrm{N} 1-\mathrm{C} 2-\mathrm{N} 3$ & $4.9(2)$ & $\mathrm{O} 7-\mathrm{C} 5-\mathrm{C} 6-\mathrm{O} 6$ & $-34.5(2)$ \\
\hline $\mathrm{O} 2-\mathrm{C} 2-\mathrm{N} 3-\mathrm{C} 4$ & $172.08(14)$ & $\mathrm{C} 4-\mathrm{C} 5-\mathrm{C} 6-\mathrm{O} 6$ & $-156.87(14)$ \\
\hline $\mathrm{N} 1-\mathrm{C} 2-\mathrm{N} 3-\mathrm{C} 4$ & $-7.4(2)$ & $\mathrm{C} 8-\mathrm{C} 5-\mathrm{C} 6-\mathrm{O} 6$ & $87.29(17)$ \\
\hline $\mathrm{C} 2-\mathrm{N} 3-\mathrm{C} 4-\mathrm{O} 4$ & $-165.40(14)$ & $\mathrm{O} 7-\mathrm{C} 5-\mathrm{C} 6-\mathrm{N} 1$ & $149.92(12)$ \\
\hline $\mathrm{C} 2-\mathrm{N} 3-\mathrm{C} 4-\mathrm{C} 5$ & $21.1(2)$ & $\mathrm{C} 4-\mathrm{C} 5-\mathrm{C} 6-\mathrm{N} 1$ & $27.56(18)$ \\
\hline $\mathrm{O} 4-\mathrm{C} 4-\mathrm{C} 5-\mathrm{O} 7$ & $35.46(19)$ & $\mathrm{C} 8-\mathrm{C} 5-\mathrm{C} 6-\mathrm{N} 1$ & $-88.28(15)$ \\
\hline $\mathrm{N} 3-\mathrm{C} 4-\mathrm{C} 5-\mathrm{O} 7$ & $-151.01(12)$ & $\mathrm{O} 7-\mathrm{C} 5-\mathrm{C} 8-\mathrm{C} 9$ & $54.47(16)$ \\
\hline $\mathrm{O} 4-\mathrm{C} 4-\mathrm{C} 5-\mathrm{C} 6$ & $156.51(14)$ & $\mathrm{C} 6-\mathrm{C} 5-\mathrm{C} 8-\mathrm{C} 9$ & $-64.75(14)$ \\
\hline $\mathrm{N} 3-\mathrm{C} 4-\mathrm{C} 5-\mathrm{C} 6$ & $-29.96(18)$ & $\mathrm{C} 4-\mathrm{C} 5-\mathrm{C} 8-\mathrm{C} 9$ & $174.60(12)$ \\
\hline
\end{tabular}


supporting information

\begin{tabular}{llll}
$\mathrm{O} 4-\mathrm{C} 4-\mathrm{C} 5-\mathrm{C} 8$ & $-85.88(16)$ & $\mathrm{C} 5-\mathrm{C} 8-\mathrm{C} 9-\mathrm{C} 10$ & $-164.80(13)$ \\
$\mathrm{N} 3-\mathrm{C} 4-\mathrm{C} 5-\mathrm{C} 8$ & $87.64(14)$ & $\mathrm{C} 2-\mathrm{C} 5-\mathrm{C} 8-\mathrm{C} 9$ & $-125.69(11)$ \\
$\mathrm{C} 2-\mathrm{N} 1-\mathrm{C} 6-\mathrm{O} 6$ & $167.92(14)$ & & \\
\hline
\end{tabular}

Hydrogen-bond geometry $\left(A,{ }^{\circ}\right)$

\begin{tabular}{lllll}
\hline$D-\mathrm{H} \cdots A$ & $D-\mathrm{H}$ & $\mathrm{H} \cdots A$ & $D \cdots A$ & $D-\mathrm{H}^{\cdots} \cdots A$ \\
\hline $\mathrm{N} 1-\mathrm{H} 1 \cdots \mathrm{O} 7^{\mathrm{i}}$ & $0.87(1)$ & $2.03(1)$ & $2.8683(17)$ & $164(2)$ \\
$\mathrm{N} 3-\mathrm{H} 3 \cdots \mathrm{O} 4^{\mathrm{ii}}$ & $0.86(1)$ & $2.00(1)$ & $2.8451(16)$ & $170(2)$ \\
$\mathrm{O} 7-\mathrm{H} 7 \cdots \mathrm{O} 2^{\mathrm{iii}}$ & $0.84(2)$ & $1.98(2)$ & $2.8055(15)$ & $169(2)$ \\
\hline
\end{tabular}

Symmetry codes: (i) $-x+3 / 2, y-1 / 2,-z+1 / 2$; (ii) $-x+1,-y+2,-z+1$; (iii) $x-1 / 2,-y+3 / 2, z-1 / 2$. 